\title{
In vitro developmental study of oil palm (Elaeis guineensis Jacq.) polyembryoids from cell suspension using scanning electron microscopy
}

\begin{abstract}
In the present study, we report the in vitro development of polyembryoids with identification of a definite stage that can be used for subsequent uniform plantlet regeneration in oil palm (Elaeis guineensis Jacq.). Induction and maturation of polyembryoids was accomplished when cell suspension culture was transferred in MS (Murashige and Skoog, Physiol Plant 15:473ï 497, 1962) semisolid medium consisting of $30 \mathrm{~g}$ Lī 1 sucrose and $3.5 \mathrm{~g}$ Lī 1 gelrite® devoid of any plant growth regulator. Growth and development of cell suspension culture into polyembryoids were assessed by stereo and scanning electron microscopy (SEM) to identify the sequential events as well as the differentiation that occur during each stage. Observations on the differentiation symptoms showed that the embryos pass through distinct morphological characteristics indicating distinctively varied stages. SEM observations indicated the development of extracellular network at an early stage of differentiation and acts as the structural marker of differentiation leading to the development of polyembryoids via formation of globular proembryo and haustorium. Eventually, a specific developmental stage comprising haustorium and torpedo-shaped structure was identified, for conservation, regeneration or multiplication, based on the embryogenic competence.
\end{abstract}

Keyword: Developmental stage; Dura $\times$ Pisifera; Extracellular matrix; Micromorphology; Multicellular suspensor; Rosette like complex; Scanning electron microscopy; Somatic embryo 